\title{
NOUVELle
}

\section{Le mosaïcisme somatique en cause dans les épilepsies neurodéveloppementales}

Théo Ribierre, Stéphanie Baulac
Institut du cerveau et de la moelle épinière (ICM), Inserm U1127, CNRS UMR 7225, Sorbonne Université, Hôpital Pitié-Salpêtrière, 47, boulevard de l'Hôpital, 75013 Paris, France.

stephanie.baulac@upmc.fr
> Les épilepsies sont des maladies neurologiques qui touchent $0,8 \%$ de la population. Elles se manifestent par la survenue spontanée et récurrente de crises traduisant une excitation synchronisée et anormale d'un groupe de neurones du cortex cérébral. Les causes de l'épilepsie sont variées: génétiques, dues à une lésion cérébrale (traumatique, tumorale, malformative, vasculaire, inflammatoire ou infectieuse), environnementales ou métaboliques.

Depuis quelques années, un intérêt croissant s'est porté sur l'étude des épilepsies focales associées à des malformations du développement cortical, en particulier les dysplasies corticales focales (DCF) [1]. Les DCF se traduisent par une lésion plus ou moins étendue selon le stade de grossesse au cours duquel elles apparaissent, qui est habituellement identifiable par imagerie médicale (TEP [tomographie par émission de positons]/IRM [imagerie de résonance magnétique]). Ces malformations dites «épileptogènes » entraînent une épilepsie focale réfractaire aux médicaments antiépileptiques. Le traitement de l'épilepsie passe alors souvent par la résection chirurgicale du foyer épileptogène à l'origine des crises, ce qui permet l'accès au tissu postopératoire à des fins de recherche. Sur le plan neuropathologique, les DCF se caractérisent par un défaut d'établissement de l'architecture du cortex cérébral au cours de l'embryogenèse et par la présence de cellules de morphologie anormale, comme les neurones dysmorphiques dans les DCF de type lla ou les cellules ballonisées dans les DCF de type IIb [2]. Récemment, l'origine génétique des DCF a été démontrée à la suite de l'identification de mutations somatiques cerveau-spécifiques dans des gènes appartenant à la voie de signalisation mTOR (mammalian target of rapamycin) [3]. II s'agit de mutations qui apparaissent durant le développement embryonnaire cérébral et dont le taux de mosaïcisme ${ }^{l}$ est corrélé à la taille de la lésion. Bien que dans la vaste majorité des cas les DCF soient sporadiques, il existe une composante héréditaire avec antécédents familiaux dans le cas de mutations touchant les gènes codant le complexe GATORI (Gap-activity towards rag complex 1 ). Ce dernier comprend trois protéines, DEPDC5 (DEP [dishevelled, EGL10 and pleckstrin] domain-containing 5), NPRL(nitrogen permease regulator-like protein)-2 et -3 , et agit comme répresseur du complexe 1 de mTOR (mTORCl) [4]. DEPDC5 est le gène le plus fréquemment muté dans les cas familiaux d'épilepsie focale associée à une DCF, et les mutations perte-de-fonction entraînent une hyperactivité de la protéine kinase $\operatorname{mTOR}[5,6]$. Toutefois, comment expliquer la présence d'un foyer dysplasique épileptogène chez certains membres d'une famille, tandis que d'autres présentent une IRM normale avec une épilepsie sans lésion apparente? Cette observation nous a conduit à envisager, selon le modèle de Knudson, la nécessité de l'accumulation de deux mutations sur les deux allèles pour l'apparition d'une lésion focale.

1 État dans lequel deux ou plusieurs populations de cellules avec des caryotypes différents coexistent dans un organisme.

\section{Développement d'un foyer dysplasique épileptogène : conséquence}

d'une mutation somatique mosaïque secondaire?

Selon le modèle du «two-hit »de Knudson proposé dans le cadre de la progression du cancer $[7,8](\rightarrow)$, nous avons formulé l'hypothèse selon laquelle l'émergence d'un foyer dysplasique épileptogène est causée par l'occurrence d'une mutation somatique mosaïque secondaire à la mutation hétérozygote constitutive du gène DEPDC5.

Afin de tester cette hypothèse, nous avons recherché des mutations somatiques spécifiques du cerveau dans un panel de gènes de la voie mTOR dans une cohorte de 10 individus présentant une FCD de type II, et pour lesquels nous avions obtenu un prélèvement postopératoire de résection cérébrale. Chez un des patients, nous avons pu identifier dans les échantillons d'ADN issus du sang et du tissu cérébral une mutation constitutive hétérozygote dans le gène DEPDC5, ainsi qu'une seconde mutation somatique présente uniquement dans I'ADN extrait du tissu cérébral. Un séquençage de grande profondeur ${ }^{2}$ a permis de confirmer que les deux mutations perte-de-fonction étaient chacune localisées sur un allèle différent, conduisant à l'inactivation bi-allélique du gène. De plus, nous avons découvert l'existence

\footnotetext{
2 Le rapport entre la longueur de l'ensemble des séquences lues mises bout à bout et la longueur du génome cible est nommé profondeur.
} 


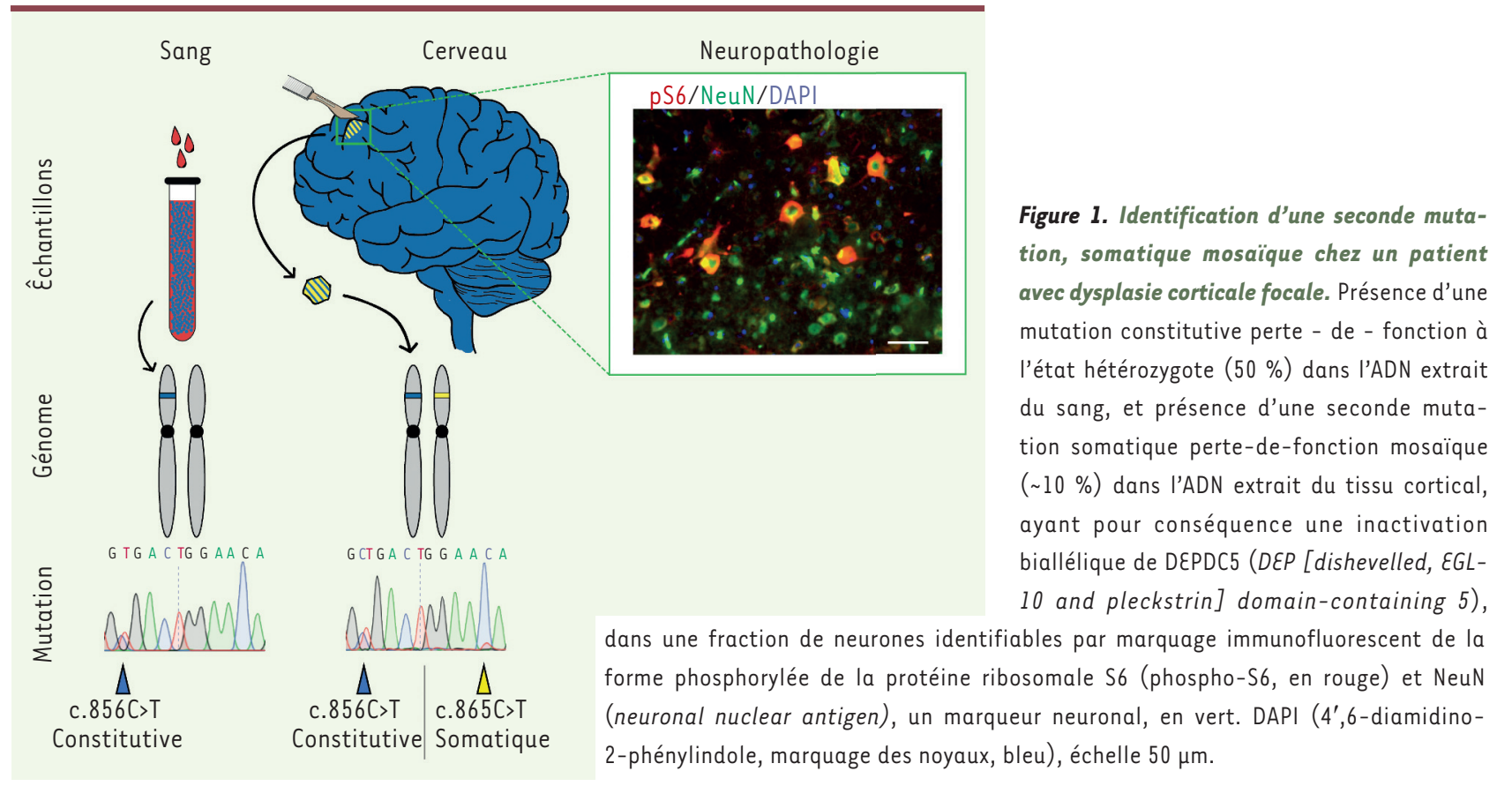

d'un gradient de mosaïsme entre la zone épileptogène d'initiation des crises (10\% de mosaïsme) et la zone épileptogène périphérique $(0,3 \%)$. Nous avons ensuite montré que l'échantillon de tissu dysplasique présentait des groupes de neurones dysmorphiques exprimant la forme phosphorylée de la protéine ribosomale S6, un substrat de la protéine kinase mTOR, témoin de l'hyperactivité de la voie mTOR dans ces cellules (Figure 1) [9].

L'occurrence d'une mutation somatique mosaïque pourrait donc expliquer la variabilité de la localisation du foyer épileptogène chez les différents membres d'une famille partageant une mutation constitutive héritée de DEPDC5.

\section{Mutations somatiques chez la souris :} un modèle de dysplasie focale corticale

La découverte d'un mécanisme mutationnel «two-hit » dans les épilepsies neurodéveloppementales nous a conduits à engendrer une délétion focale mosaïque de Dedpc5 au cours de l'embryogenèse chez la souris. Nous avons induit chez la souris Swiss-Webster une inactivation mosaïque biallélique de Depdc5 au stade gestationnel $\varepsilon 14,5$, dans les progéniteurs de neurones pyramidaux excitateurs, affectant environ $2 \%$ des cellules d'un cerveau embryonnaire en combinant deux techniques innovantes : l'électroporation in utero et l'édition génomique CRISPRCas9 afin d'éteindre (knockout) le gène de manière focale et mosaïque (Depdc5 ${ }^{\text {fKO }}$ ). Nous avons tout d'abord remarqué un retard de la migration neuronale dans le cortex des embryons, qui persistait chez les souris adultes. Au sein de la zone électroporée, nous avons observé la présence de neurones volumineux et de cellules ballonnisées, avec un immunomarquage de phospho-S6, indiquant I'hyperactivation de la voie mTOR. L'enregistrement continu par vidéoélectroencéphalogramme des souris Depdc $5^{\mathrm{fKO}}$ a alors mis en évidence la manifestation de crises spontanées d'épilepsie chez $30 \%$ des souris adultes. Ces crises surviennent à l'âge adulte et sont suivies par la mort subite de l'animal, ce qui rappelle une comorbidité tragique de la pathologie : la mort subite et inexpliquée en épilepsie (SUDEP) [6]. Nous avons ensuite recherché de possibles altérations morphologiques et électrophysiologiques des cellules pyramidales électroporées de la couche corticale III, deux semaines en amont de l'âge d'apparition des crises. Ces neu- rones présentaient une augmentation significative du nombre d'embranchements dendritiques, une projection plus dispersée et complexe du plumeau apical dendritique, ainsi qu'une hypertrophie des dendrites et de leurs épines. Enfin, bien que les propriétés intrinsèques des neurones modifiés par CRISPR-Cas9 aient mis en évidence une hypoexcitabilité vraisemblablement due à l'augmentation de la capacitance et à la diminution de la résistance membranaires, l'amplitude des courants spontanés excitateurs s'est révélée significativement plus importante. Ces altérations électrophysiologiques pourraient être une conséquence de l'hypertrophie cellulaire en combinaison avec une altération de l'expression de récepteurs glutamatergiques postsynaptiques [8].

Ce nouveau modèle murin récapitule plusieurs aspects de la pathologie avec l'apparition de crises d'épilepsies spontanées et la présence de cellules morphologiquement anormales présentant une hyperactivation de la kinase mTOR.

\section{De nouvelles perspectives thérapeutiques}

Notre étude souligne le rôle fondamental des mutations cérébrales somatiques 
dans l'émergence de pathologies neurodéveloppementales. Nous apportons la preuve de l'existence d'une inactivation biallélique (constitutive hétérozygote et somatique mosaïque) dans un gène codant un répresseur de la voie mTORCl responsable du développement de malformation corticale. Cela met en évidence un mécanisme différent des mutations somatiques mosaïques gainde-fonction sur un seul allèle du gène $M T O R$, les plus fréquemment retrouvées dans les cas sporadiques de DCF [10].

La compréhension des mécanismes génétiques responsables du développement de malformations corticales associées à une épilepsie focale, ainsi que l'établissement d'un modèle murin pertinent, permettent à présent de développer de nouvelles stratégies thérapeutiques pour ces patients réfractaires aux traitements actuels. Ces stratégies sont essentielles dans le panorama chirurgical actuel de l'épilepsie, où les malformations corticales du développement prennent une part grandissante [11]. $\diamond$ A second-hit somatic mutation drives neurodevelopmental epilepsy

\section{LIENS D'INTÉREิT}

Les auteurs déclarent n'avoir aucun lien d'intérêt concernant les données publiées dans cet article.

\section{RÉFÉRENCES}

1. Iffland PH $2^{\text {nd }}$, Crino PB. Focal cortical dysplasia gene mutations, cell signaling, and therapeutic implications. Annu Rev Pathol 2017 ; 12 : 547-71.

2. Blumcke I, Thom M, Aronica $\varepsilon$, et al. The clinicopathologic spectrum of focal cortical dysplasias : a consensus classification proposed by an ad hoc task force of the ILAE diagnostic methods commission. Epilepsia 2011 ; 52 : 158-74.

3. Marsan $\varepsilon$, Baulac $S$. Mechanistic target of rapamycin (mTOR) pathway, focal cortical dysplasia and epilepsy. Neuropathol Appl Neurobiol 2018 ; 44 : 6-17.
4. Bar-Peled L, Chantranupong L, Cherniack AD, et al. A tumor suppressor complex with GAP activity for the Rag GTPases that signal amino acid sufficiency to mTORC1. Science $2013 ; 340$ : 1100-6.

5. Ishida S, Picard F, Rudolf G, et al. Mutations of DEPDC5 cause autosomal dominant focal epilepsies. Nat Genet $2013 ; 45$ : 552-5.

6. Baldassari S, Picard F, Verbeek B, et al. The landscape of epilepsy-related GATORl variants. Genet Med 2018 ; $21: 398-408$.

7. Knudson AG, Jr. Mutation and cancer : statistical study of retinoblastoma. Proc Natl Acad Sci USA $1971 ; 68: 820-3$.

8. Magerus-Chatinet $A$, Rieux-Laucat F. Le modèle du two-hit de Knudson s'applique aux maladies autoimmunes. L'exemple du syndrome lymphoproliferatif avec auto-immunité (ALPS). Med Sci (Paris) 2011 . $27: 107-9$

9. Ribierre T, Deleuze C, Bacq A, et al. Second-hit mosaic mutation in mTORCl repressor DEPDC5 causes focal cortical dysplasia-associated epilepsy. J Clin Invest $2018 ; 128: 2452-8$.

10. Lim JS, Kim WI, Kang HC, et al. Brain somatic mutations in MTOR cause focal cortical dysplasia type II leading to intractable epilepsy. Nat Med 2015 ; 21 : 395-400.

11. French J, Friedman D. The evolving landscape of epilepsy neuropathology. Lancet Neurol 2018; 17 : 202-3. 